\title{
Systems biology and the discovery of diagnostic biomarkers
}

\author{
Kai Wang ${ }^{\mathrm{a}, *}$, Inyoul Lee ${ }^{\mathrm{a}}$, George Carlson ${ }^{\mathrm{b}}$, Leroy Hood ${ }^{\mathrm{a}}$ and David Galas ${ }^{\mathrm{a}}$ \\ ${ }^{\mathrm{a}}$ Institute for Systems Biology, WA, USA \\ ${ }^{\mathrm{b}}$ McLaughlin Research Institute, Great Falls, MT, USA
}

\begin{abstract}
Systems biology is an approach to the science that views biology as an information science, studies biological systems as a whole and their interactions with the environment. This approach, for the reasons described here, has particular power in the search for informative diagnostic biomarkers of diseases because it focuses on the fundamental causes and keys on the identification and understanding of disease- perturbed molecular networks. In this review, we describe some recent developments that have used systems biology to address complex diseases - prion disease and drug induced liver injury- and use these as examples to illustrate the importance of understanding network structure and dynamics. The knowledge of network dynamics through in vitro experimental perturbation and modeling allows us to determine the state of the networks, to identify molecular correlates, and to derive new disease treatment approaches to reverse the pathology or prevent its progress into a more severe state through the manipulation of network states. This general approach, including diagnostics and therapeutics, is becoming known as systems medicine.
\end{abstract}

Keywords: Systems biology, biomarkers, systems medicine, prion disease, drug induced liver injury, microRNA, organ-specific proteins

\section{Systems biology and systems medicine}

An approach to analyzing biological systems, which we call systems biology, has emerged over the past 10 years or so - an approach that looks at biology as an information science, studies systems as a whole and their interactions with the environment. This approach recognizes that biological information in living systems is captured, transmitted, modulated, and integrated by biological networks made up of molecular components as well as cells. This approach differs from early "systems approaches to biology" in that it attempts both a bottom-up approach (using large molecular datasets) and a top-down approach (using computational modeling and simulations) where there is an attempt to trace observations of complex phenotypes back to information encoded in the genome.

\footnotetext{
*Corresponding author. Tel.: +1 206732 1336; Fax: +1 206732 1253; E-mail: kwang@systemsbiology.org.
}

Two fundamental types of biological information, the digital information of the genome and interacting information from the environment, are both contributors to the fundamental mechanisms of life - evolution, development, physiological responses, aging and the onset and progression of disease [1]. New measurement and visualization technologies together with powerful computational and modeling tools have transformed biology by making possible the five features of contemporary systems biology: 1) measuring and quantifying various types of global biological information (e.g., sequence the entire genome, identify and quantify gut microbiome, measure the expression level of all the genes, proteins, metabolites, etc.); 2) integrating information at different levels (DNA, RNA, protein, cells etc.) to understand the interactions between the system and its environment, thereby deciphering the biological responses; 3) studying the $d y$ namical changes of all biological systems (e.g. networks) as they capture, transmit, integrate, adapt and 
respond to the environment; 4) modeling the biological system through the integration of global and dynamic data from a variety of information hierarchies; and 5) testing and improving the models through a series of iterative prediction and comparison steps. Once the models accurately fit the data from biological system responses, they can be used to predict the responses to perturbations (e.g. for identifying likely underlying causes for disease, for designing treatment strategies, or for developing preventative measure).

Diagnostic methods in past medical practice have been pauci-parameter in nature - usually measuring just a single parameter in attempts to decipher a specific disease condition (e.g. to cite a known marker, useful but badly flawed as a diagnostic, prostate specific antigen for prostate cancer [2,3]); therefore, the ability to accurately differentiate health and disease, or further identify different disease categories/subtypes has been extremely limited. The transformation in biology through systems biology will enable a new medical discipline - systems medicine - to begin to emerge in the next few years. The central premise of systems medicine is that clinically detectable molecular fingerprints resulting from disease-perturbed biological networks will be used to detect and stratify various pathological conditions. Disease associated molecular fingerprints will eventually be used to group individuals into sub-populations based on variations in genetic makeup of the population that affects disease progression. The key to this revolution lies in harnessing the power of network models of core biological processes learned through systems biology methods, combined with vast amounts of diverse molecular information generated from patient samples. Rapid advances in measurement technologies, including genome sequencing, gene expression, visualization and computational technologies are changing the face of patient studies. Molecular "fingerprints" associated with specific pathological processes (panel of biomarkers) can be composed of many different types of biomolecules, including proteins [4], DNA [5], RNA [6] or micoRNA (miRNA) [7], and metabolites [8], as well as informative, post-translational modifications to these molecules, such as protein phosphorylation [9] and glycosylation $[10,11]$. Accurate multi-parameter analyses will be the key to identifying, assessing, and tracking these molecular patterns that reflect disease-perturbed networks.

\section{Using systems biology to decipher complex pathology}

\subsection{Prion disease}

To demonstrate the power of systems biology in understanding complex diseases, we adapted a prion disease mouse model and conducted a comprehensive transcriptomic analysis of the brain through the onset of disease progression. Prion disease is a complex disease that encompasses Kuru, Creutzfeldt-Jakob disease (CJD), Gerstmann-Sträussler-Scheinker syndrome (GSS), and fatal insomnia in humans; scrapie in sheep and goats; and bovine spongiform encephalopathy in cattle [12-14]. All of these diseases are believed to arise from $\mathrm{PrP}^{\mathrm{sc}}$ - misfolding of the normal prion protein $\left(\mathrm{PrP}^{\mathrm{C}}\right)$ resulting severe brain abnormalities including spongiform degeneration of the brain, neuron loss, and astrocytic gliosis [15]. In studying prion diseases, it presents several unique challenges including 1) the etiology of the disease, 2) the mechanistic details of prion infection and replication [16], 3) pathogenic differences among different infectious prion molecules [17,18], and 4) lack of accurate blood-based detection and monitoring methods.

To understand the etiology and onset of the disease at a molecular level, to identify novel intervening targets and to discover diseases-associated molecular fingerprints for the detection and monitoring of the progression of the diseases, we applied a systems biology approach to analyze the dynamic onset of the disease after inoculating infectious $\mathrm{PrP}^{\mathrm{sc}}$ protein at the transcriptome level of brain tissues from several different strains of mice. From a collaborative study between the Institute for Systems Biology and the McLaughlin Research Institute, we identified a series of interacting networks involving prion accumulation, glial cell activation, synapse degeneration and nerve cell death that were significantly perturbed by the $\operatorname{PrP}^{\mathrm{sc}}$ protein during the span of disease progression - from disease initiation to death [19]. The most important finding was that the initial molecular network changes occur well before any detectable clinical sign of disease (Fig. 2).

These dynamically changing $\mathrm{PrP}^{\mathrm{sc}}$-perturbed networks lead to two important conclusions. First, some significant network nodal points (e.g. transcripts) change before the related clinical or histological changes are apparent. Therefore, labeled molecular probes such as antibodies that are specific to the changing nodes or the biological processes they regulate could be used for in vivo imaging diagnostics even 


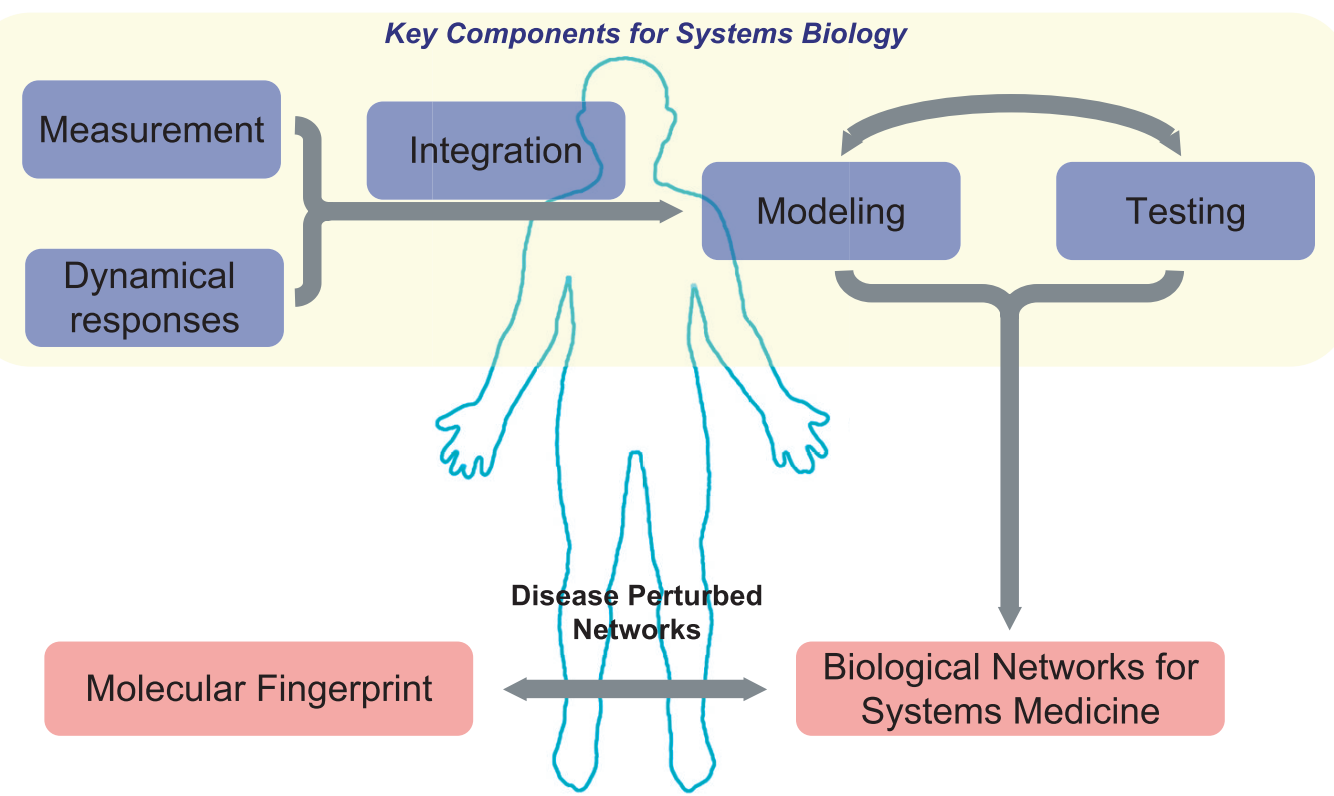

Fig. 1. The key components of systems biology.

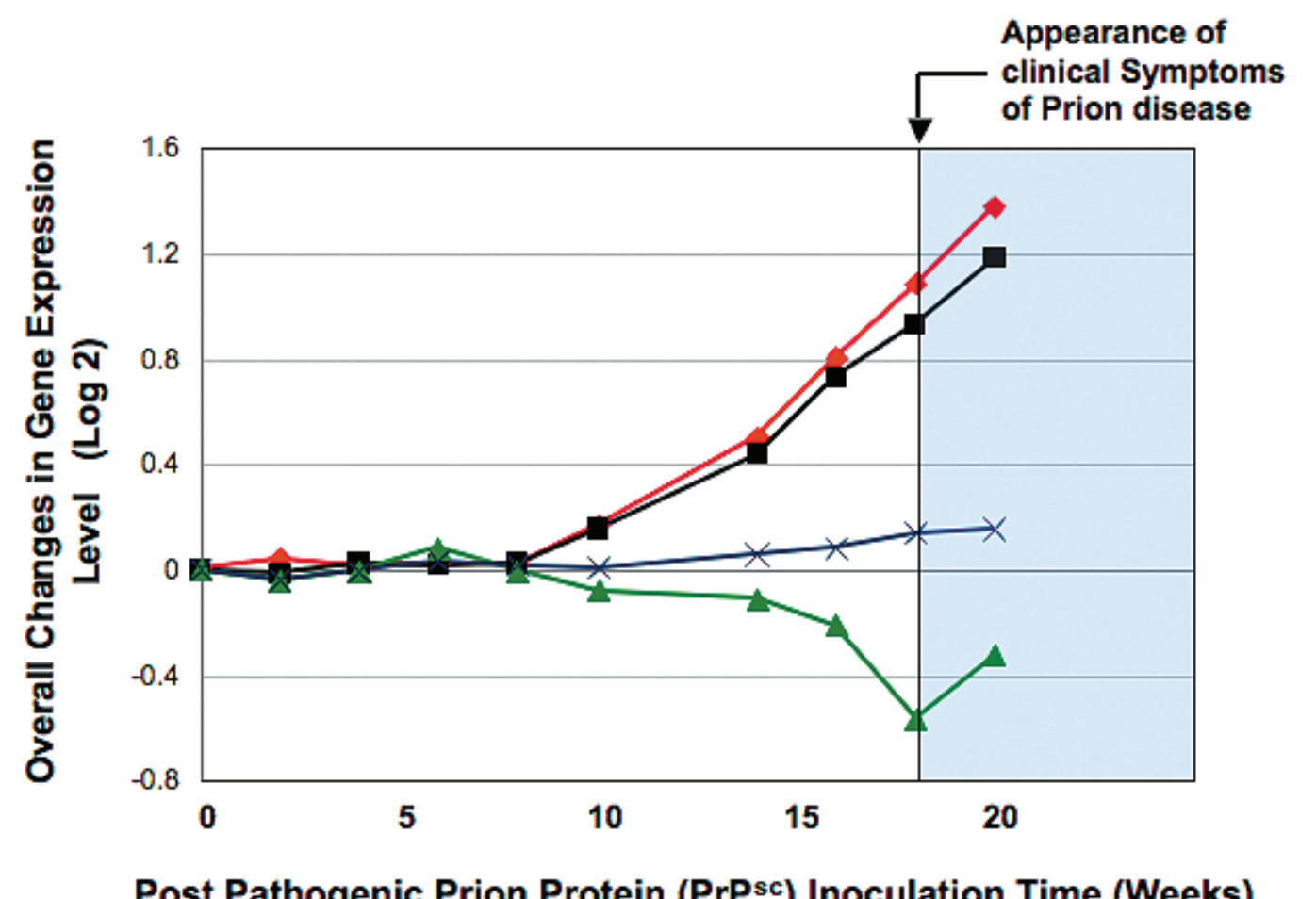

$\longrightarrow$ Microglial activation $\rightarrow$ PrPrs accumulation $\rightarrow$ Neurodegeneration $\rightarrow$ Neuronal cell death

Fig. 2. The key sub-networks affected by pathogenic prion inoculation. The mean of relative changes of all genes involved in each network were plotted. The value of relative changes compared to control is indicated on the Y-axis (in $\log 2$ value) while the $\mathrm{X}$-axis is the time post pathogenic prion inoculation (in weeks). The appearance of clinical symptoms of the disease is around 18 weeks as indicated on the figure (highlighted in blue). 
Table 1

Shared Subnetworks, pathways and modules among neurodegenerative diseases

\begin{tabular}{llllll}
\hline Subnetwork & Pathway and module & Prion ref & AD ref & HD ref & PD ref \\
\hline Glial activation & Complement activation & $\mathrm{Hw}$ & $\mathrm{Bl}, \mathrm{K}, \mathrm{Z}$ & $\mathrm{Bon}$ & $\mathrm{Bon}$ \\
& Reactive astrogliosis & $\mathrm{Hw}$ & $\mathrm{N}, \mathrm{Z}, \mathrm{Mr}$ & $\mathrm{Ml}$ & $\mathrm{Te}$ \\
& Microglia activation & $\mathrm{Hw}$ & $\mathrm{S}, \mathrm{Me}, \mathrm{Z}, \mathrm{Mr}$ & $\mathrm{Ml}$ & $\mathrm{Te}$ \\
& Cytokinesis, Chemokinesis & $\mathrm{Hw}$ & $\mathrm{Bl}, \mathrm{S}, \mathrm{Mr}$ & $\mathrm{Ho}$ & $\mathrm{M} 2, \mathrm{Te}$ \\
Synapse degeneration & Synaptic transmission & $\mathrm{Hw}$ & $\mathrm{Bl}$ & $\mathrm{W}$ & $\mathrm{Bos}, \mathrm{Si}$ \\
& Neurotransmitter & $\mathrm{Hw}$ & $\mathrm{Bl}$ & $\mathrm{Ho}$ & $\mathrm{Bos}, \mathrm{Si}$ \\
& Calcium signaling & $\mathrm{Hw}$ & $\mathrm{Bl}, \mathrm{N}$ & $\mathrm{Ho}, \mathrm{D}$ & $\mathrm{Bos}, \mathrm{Si}$ \\
& Cation trasport & $\mathrm{Hw}$ & $\mathrm{Bl}$ & $\mathrm{D}$ & $\mathrm{Bos}$ \\
& Mitochondria dysfunction & $\mathrm{Hw}$ & $\mathrm{R}, \mathrm{Yao}$ & $\mathrm{Bossy}$ & $\mathrm{Ha}, \mathrm{Si}$ \\
& Autophage regulation & $\mathrm{Hw}$ & $\mathrm{Ct}, \mathrm{Bol}$ & $\mathrm{Ho}, \mathrm{Sa}$ & $\mathrm{O}$ \\
& Ubiquitination & $\mathrm{Hw}$ & $\mathrm{Bl}$ & $\mathrm{W}$ & $\mathrm{Ha}, \mathrm{Bos}, \mathrm{Si}$ \\
& Apoptosis & $\mathrm{Hw}$ & $\mathrm{Bl}, \mathrm{R}, \mathrm{T} 1$ & $\mathrm{I}$ & $\mathrm{Ha}, \mathrm{Si}$ \\
\hline
\end{tabular}

$\mathrm{AD}=$ Alzheimer's Disease; HD = Huntington's disease; $\mathrm{PD}=$ Parkinson disease.

Hw = Hwang et al., 2009.

B1 = Blalock 2004; K = Kolev et al., 2009; $\mathrm{Z}=$ Zhou et al., 2008; $\mathrm{N}=$ Noris et al., 2005.

$\mathrm{Mr}=$ Mrak and Griffin 2005; S = Shie et al., 2009; Me = Meda et al., 2001.

$\mathrm{Ct}=$ Cataldo et al., 2008; $\mathrm{R}=$ Reddy et al., 2004; Y = Yao et al.,2009.

Bol = Boland et al., 2008; T1 = Tang et al.,2008.

Bon = Bonifati and Kishore, 2006; M1 = Maragakis and Rothstein 2006.

Ho = Hodges 2007; W = Wang et al., 2007; D= Desplats et al., 2006.

$\mathrm{I}=$ Inagaki et al., 2008; Bossy = Bossy et al., 2008; Sa = Sarkar and Rubinsztein, 2008.

Bos = Bossers et al., 2009; $\mathrm{Si}=$ Simunovic et al., 2009; Te = Teismann and Schulz, 2004.

M2 = Mandel et al., 2000; O= Olzmann et al., 2008.

$\mathrm{Ha}=$ Hauser et al., 2005.

before symptoms arise, as has already been shown in patients [20,21]. Alternatively, if some of these altered transcripts encode secreted proteins, they could provide readily accessible in vitro diagnostic blood markers for early disease detection. Second, many of the sub-networks of proteins that change during the onset of disease affect changes in phenotypic traits that are consistent with the pathology of the disease. A core of 333 perturbed genes appear to encode the heart of prion-disease response - that is, when mapped onto four major protein networks (prion accumulation, glial cell activation, synapse degeneration and nerve cell death) they explain virtually every known aspect of the prion pathology. In addition, new modules such as iron homeostasis, leukocyte extravasation and prostaglandin metabolism have been identified that may encode aspects of this disease that were not previously known. Many of the 333 prion-perturbed genes identified in our prion mouse models also are seen in other neurodegenerative disorders such as Alzheimer's disease. Moreover, three of the four perturbed networks (glial cell activation, synapse degeneration and nerve cell death) that we identified in the prion disease model are also evident in other human neurodegenerative diseases - Alzheimer's disease [22,21-33], Huntington's disease [34-41], and Parkinson's disease [34,4246]. Therefore, we may find common pathological processes involved in different neurodegenerative diseases and provide fundamental insights of brain pathology through the prion model system despite its diverse etiology (Table 1).

Moreover, during the progression of prion disease, many transcripts undergo significant changes in expression levels well before the appearance of any clinical signs. Many of these potential early-disease signatures are predicted to encode secreted proteins and some of these proteins such as Serpina3n, Ctss, Abca1 have been robustly detected by mass spectroscopy in serum or plasma (http://www.peptideatlas.org) and therefore represent potential blood-based protein biomarkers for early detection of prion disease.

\subsection{Liver toxicity}

Drug induced liver injury (DILI) is the key reason for compounds failing in clinical development and is the most common cause for withdrawal of drugs from the market. It is not only a significant financial burden for pharmaceutical companies, but also a serious health care problem. Results from population studies clearly indicate significant variability among individuals toward sensitivity as well as efficacy on drugs such as Warfarin, Paclitaxel, Morphine, Lipitor and Acetaminophen $[48,49]$. In certain cases, genetic loci that are associated with such differences, such as Oprm (u-opioid receptor) and Htr1b (serotonin-1B receptor) 
in morphine sensitivity have been identified through pharmacogenetic and pharmacogenomic studies either in the human population or in animal models [50,51]. The complexity and difficulty of conducting an accurate drug safety evaluation and efficacy assessment in a genetically diverse population evidently suggests the need for a new approach for such processes. A recent report identifying 26 common biomarkers for acetaminophen overdose induced liver injury based on a panel of 36 genetic diverse inbred mouse strains clearly supports the power of including data from different genetic background in the experimental model [52].

Besides studying diseases provoked by infectious agents, such as the pathogenic prion protein, at the Institute for Systems Biology, we have also made a significant effort in using systems biology to understand the fundamental biology associated with acetaminophen induced liver injury in mouse - a well studied model system for DILI. We conducted comprehensive time-course analyses on protein and RNA in blood and liver tissues obtained from animal models. Networks associated with inflammatory responses, drug metabolism, and tissue regeneration are unequivocally involved in the progression of liver injury induced by acetaminophen overdose based on our data. In addition, we also observed changes in blood levels of a number of proteins originated from the liver ( $\mathrm{Hu}$, Sun and Hood, personal communication). Most of these proteins are liver-specific and involved in networks associated with general metabolic activities, such as carbohydrate and amino acid metabolism. The alterations in the levels of some of these proteins in plasma are far more sensitive than the current serological markers and deserve further evaluation in the future.

MicroRNAs (miRNA) are small regulatory, noncoding RNAs. It is believed that miRNAs primarily affect the stability of mRNA and/or the initiation and progression of protein translation. To date, there are about 500 different microRNA species identified in mouse and about 700 miRNAs in human, some of which display restricted tissue expression profiles similar to $\mathrm{mR}$ NA. Even though the biological function of miRNA is yet to be fully understood, it has been shown that the levels of specific miRNAs in tissues correlate well with the pathological development of several different diseases [53-55]. In our acetaminophen overdose induced liver injury model, we also observed changes in a number of miRNAs in the liver tissue, but to our surprise, a fair number of cell-free miRNAs are in the plasma and some of their levels are closely related to the status of liver injury [56]. One of the most intriguing miR-
NA species in this population is the liver-specific miR122. Its level rose much earlier than the traditional liver injury markers, the serum alanine aminotransferase (ALT) (Fig. 3). We also observe similar changes in a limited number of plasma samples from human individuals suffering from acetaminophen overdose. Lower complexity, no post-processing modification, synthetic high affinity "capture" reagents, tissue restricted expression profile, and "amplifiable" signals make the extracellular miRNAs good candidates as biomarkers to reflect various physiopathological conditions in the body. Combining these organ-specific miRNAs with proteins will likely lead to highly informative panels to identify and stratify various diseases in the future.

Three important conclusions from our prion and DILI systems biology studies are: 1) the power of using multiple strains of mice to stratify the diverse genetic variations in human population - this approach also reduced the experimental and environmental noise and identified the core/common set of genes involved in pathology, 2) the necessity of using time course data to identify and verify the dynamical changes of disease-perturbed networks, and 3) disease-associated molecules in the blood can be used to detect and monitor the pathology in distant organs. Biological fluids such as urine and blood have been used in diagnostic applications for many years due to their non-invasive nature and can be adapted to high throughput screening format.

\section{Blood - the information highway in the body - finding blood biomarkers}

The blood is in close contact with every organ in the body to convey information, deliver nutrients, carry waste, and survey the homeostatic status of tissues. It is the information highway in the body as the Internet is in contemporary society. Therefore, the blood will be an extremely informative window to assess multi-organ biological responses to environmental stimulations and to detect specific markers associated with developing pathology in the body. However, attempts to identify new biomarkers in blood for various diseases have thus far proved to be surprisingly disappointing. We believe this is largely because the unprecedented molecular complexity, the diverse origin of the biomolecules in different organs and cell types, the stability or instability of the molecules, and the low abundance of many of the molecules of interest in the blood [1,57-60]. The major difficulty with this approach is that because of 
A)

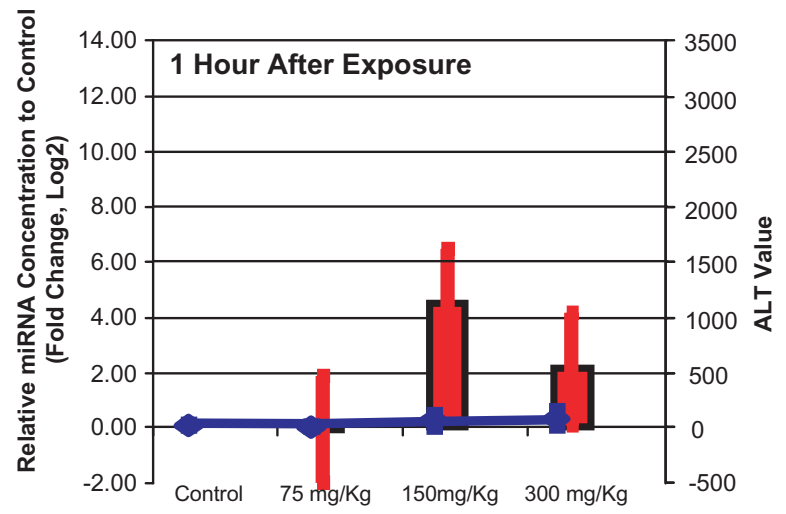

B)

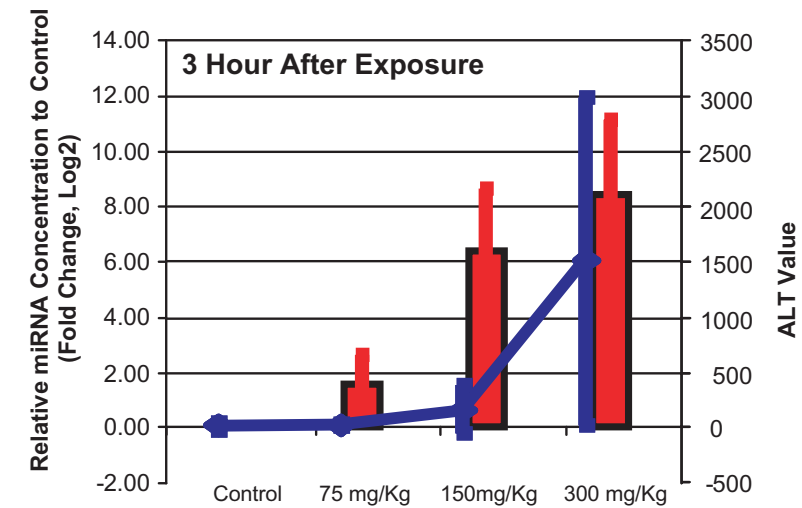

Fig. 3. The level of circulating miRNA is more sensitive marker for drug induced liver injury. The comparison between the levels of mir-122 (red bars) and ALT (blue line) in plasma samples collected from mice 1 (A) and 3 hours (B) after exposure to different doses of acetaminophen (indicated on X-axis). The relative change of miRNA expression levels (ratio in log 2 compare to control) is indicated on the left side of the figure and the scale of ALT level is on the right. The values of miRNA fold change and ALT levels are the average of 4 independent samples from each time point and the standard derivations are shown as error bars.

the high degree of undeciphered complexity, markers identified as potential correlates with disease states can change in unpredictable ways since many are expressed and released by multiple organs that may respond differently to disease and a variety of environmental perturbations. Hence, diagnostic non-specificity arises due to the uncertainty regarding the tissue of origin of these markers (high noise levels that are largely uncharacterized). The solution to this dilemma that we have pursued is to identify and focus on organ-specific biomarkers (majority of the message produced normally by only one organ) whose changes therefore most likely reflect changes in the selected organ itself.

Using an organ-specific protein/RNA fingerprint for diagnostics is one of the key information-based approaches that lies at the heart of the systems medicine that will emerge over the next few years. The idea is that pathology arises from dynamically disease-perturbed networks (can be identified using systems biology to study patient samples and model systems), that the diseased organs will release molecules including proteins, RNAs, and other biomolecules into the blood, and that if these molecules are encoded by/associated with disease-perturbed networks then their levels in the blood might be altered in a manner that reflects the specific nature of the disease. If enough of these organspecific or network-specific biomolecules in blood are sampled, the results will represent a global survey of the status of many different biological networks in the organ of interest and will provide sufficient diagnostic information for any disease.

\section{Conclusion and outlook for systems medicine}

The basic question of how systems biology approaches facilitate the discovery of informative biomarkers is answered by extracting four key points from the above discussion and description of examples. 1. Networks are perturbed from their normal states during disease. 2. Organ-specific components of these networks can be used to infer the state of specific networks in the targeted organs. 3. Panels of multiple blood markers including organ-specific and process-specific markers in the blood can provide abundant information about what is taking place in the affected organs and cell types. 4. The selected proteins or RNAs that are released into the blood provide assessment of the state of and future evolution of perturbed networks.

When it becomes possible to monitor the precise state of the networks or key sub-networks in a tissue or organ by the levels of a spectrum of biomarkers in the blood, the current disease diagnostics and medical practice will have to shift in a profound and fundamental way. The future systems medicine approach to disease treatment is based on the ability to identify and assess the state of the perturbed networks as well as the dynamic evolution of such networks under a variety of perturbations. This will allow in turn, the choice of the right intervention to abrogate the progression of the pathology. Knowing the dynamics of the networks and their interactions, we will be able to define the state of the pathological process at the biological network level, predict the progression of the pathology based on network interactions, and derive interventions to restore 


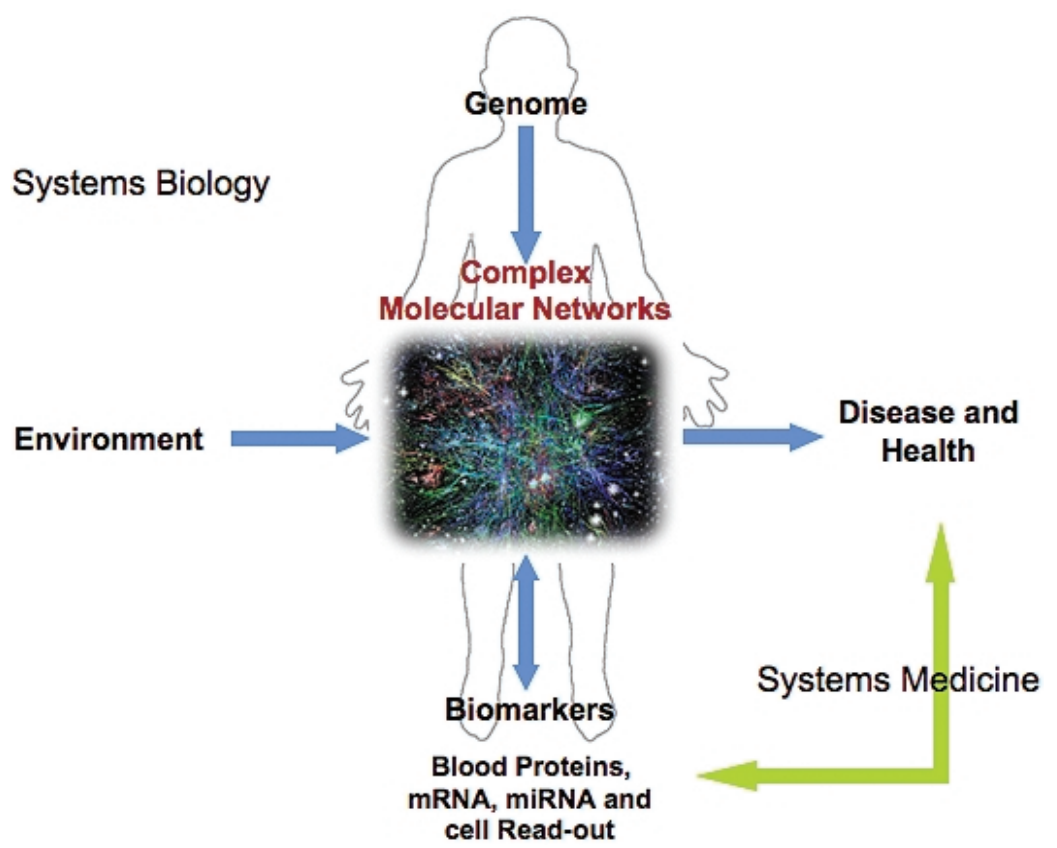

Fig. 4. Understanding the complex molecular network is the center of systems biology and systems medicine.

the networks to the normal state. A key point here is that knowing the precise states and the way in which these states evolve at the molecular network level, will open the door to being able to specifically intervene or modify key sub-networks to prevent the predicted evolution of the pathology. The therapeutic component of systems medicine then, in which we infer network states from biomarker measurements and intervene to halt and reverse the networks progress into an undesired state, depends on our ability to: 1) precisely infer network state from the results of assessing the levels of a panel of informative, diagnostic biomarkers in the blood, and 2) specifically manipulate a network to avoid or revert the pathology. The later is a very complex issue and beyond the ability of our current understanding of networks to accomplish. It is clear, however, that this is possible in principle, and we fully expect that it will be accomplished, at least in some situations, within the next decade.

Based on the development of systems biology in the past decade, the future of systems medicine is predicted to unfold in the following ways. Blood biomarkers will be discovered that correlate with, and are causally related to, key pathologies. The underlying network states will be deciphered and the pathologies specifically associated with key networks and sub-networks states will be identified. This advance in deciphering of the molecular complexity and seeing through the phys- iological noise will enable a revolution in diagnostics comprehensive understanding of pathology and its progression at molecular levels through the qualitative and quantitative measurement of blood biomarkers. The next step will require a deep understanding of network dynamics and of the effects of a range of potential perturbations. Systems medicine will then evolve from diagnostic based medicine into a diagnostic-therapeutic based systems medicine through identifying and manipulating the state of perturbed biological networks.

In addition to the difficulty in acquiring and understanding the necessary depth and dynamics of network information associated with pathological processes, we will be facing challenges, both practical and fundamental. Practical difficulties will arise from such issues as the noise that derives from diurnal variations of physiology and a wide range of affecting environmental factors (including pathogens, diet, exercise and exposure to stresses.) In addition, the genetic differences among humans that can modify the networks and affect their function and responses to perturbations will further complicate the association between network state and specific pathology. The use of systems biology to integrate genetic and environmental factors to fully understand the network structure and dynamics is therefore going to be a critical step to reach the full potential of systems medicine (Fig. 4).

The future of systems medicine will ultimately be the application of our understanding of the integrat- 
ed dynamical responses of various molecular networks that determine the critical states of the body. When we fully understand the key biological systems involved in pathologies, we will be able to quickly assess and correct, modify, or adjust the molecular network responses accordingly to revert the pathological state or to prevent the progression into a more severe state. This future is still out of our reach, but the path to this future is becoming increasingly clear.

\section{Acknowledgements}

We would like to acknowledge Drs. Alton Etheridge, Ji-Hoon Cho, Zhiyuan Hu, Hyuntae Yoo, and Bingyun Sun, for their critical reading of the manuscript and stimulating discussion on blood biomarkers. This work was supported by the ISB-University of Luxemburg program, Systems Biology Center grant (GM076547) and a prion disease grant (NS41997) from NIH, and research contracts from the Battelle Biology and Health Science Initiative (Battelle OP46250) and the Department of Defense (W911SR-07-C-0101 and HDTRA 1-08-C-0023).

\section{References}

[1] L. Hood et al., Systems biology and new technologies enable predictive and preventative medicine, Science 306(5696) (2004), 640-643.

[2] R. Mazzucchelli et al., Prostate tissue and serum markers, $A d v$ Clin Path 4(3) (2000), 111-120.

[3] W.W. Hochreiter, The issue of prostate cancer evaluation in men with elevated prostate-specific antigen and chronic prostatitis, Andrologia 40(2) (2008), 130-133.

[4] X. Wang et al., Autoantibody signatures in prostate cancer, $N$ Engl J Med 353(12) (2005), 1224-1235.

[5] E. Papadopoulou et al., Cell-free DNA and RNA in plasma as a new molecular marker for prostate and breast cancer, Ann $N$ Y Acad Sci 1075 (2006), 235-243.

[6] K.D. Sorensen and T.F. Orntoft, Discovery of prostate cancer biomarkers by microarray gene expression profiling, Expert Rev Mol Diagn 10(1), 49-64.

[7] C.R. Scherzer et al., Molecular markers of early Parkinson's disease based on gene expression in blood, Proc Natl Acad Sci U S A, 2007.

[8] K.S. Solanky et al., Application of biofluid $1 \mathrm{H}$ nuclear magnetic resonance-based metabonomic techniques for the analysis of the biochemical effects of dietary isoflavones on human plasma profile, Anal Biochem 323(2) (2003), 197-204.

[9] A. Deguchi et al., Vasodilator-stimulated phosphoprotein (VASP) phosphorylation provides a biomarker for the action of exisulind and related agents that activate protein kinase $\mathrm{G}$, Mol Cancer Ther 1(10) (2002), 803-809.

[10] P.A. Norton et al., N-linked glycosylation of the liver cancer biomarker GP73, J Cell Biochem 104(1) (2008), 136-149.
[11] P.M. Drake et al., Sweetening the Pot: Adding Glycosylation to the Biomarker Discovery Equation, Clin Chem, 2009.

[12] S.B. Prusiner, Prions, Proc Natl Acad Sci U S A 95(23) (1998), 13363-13383.

[13] S.B. Prusiner, Prion diseases and the BSE crisis, Science 278(5336) (1997), 245-251.

[14] S.B. Prusiner, An introduction to Prion Biology and Disease, in Prion Biology and Diseases. Cold Spring Harbor Laboratory Press, 2003, pp. 1-88.

[15] S.J. DeArmond and S.B. Prusiner, Etiology and pathogenesis of prion diseases, Am J Pathol 146(4) (1995), 785-811.

[16] D. Westaway and G.A. Carlson, Mammalian prion proteins: enigma, variation and vaccination, Trends Biochem Sci 27(6) (2002), 301-307.

[17] S.J. DeArmond et al., Three scrapie prion isolates exhibit different accumulation patterns of the prion protein scrapie isoform, Proc Natl Acad Sci U S A 90(14) (1993), 6449-6453.

[18] M. Bruce et al., Transmission of bovine spongiform encephalopathy and scrapie to mice: strain variation and the species barrier, Philosophical Transactions 343 (1994), 405411.

[19] D. Hwang et al., A systems approach to prion disease, Mol Syst Biol 5 (2009), 252.

[20] A.F. Keller, M. Gravel and J. Kriz, Live imaging of amyotrophic lateral sclerosis pathogenesis: disease onset is characterized by marked induction of GFAP in Schwann cells, Glia 57(10) (2009), 1130-1142.

[21] M.E. Phelps, Inaugural article: positron emission tomography provides molecular imaging of biological processes, Proc Natl Acad Sci U S A 97(16) (2000), 9226-9233.

[22] B. Boland et al., Autophagy induction and autophagosome clearance in neurons: relationship to autophagic pathology in Alzheimer's disease, J Neurosci 28(27) (2008), 6926-6937.

[23] A.M. Cataldo et al., Down syndrome fibroblast model of Alzheimer-related endosome pathology: accelerated endocytosis promotes late endocytic defects, Am J Pathol 173(2) (2008), 370-384.

[24] L. Meda, P. Baron and G. Scarlato, Glial activation in Alzheimer's disease: the role of Abeta and its associated proteins, Neurobiol Aging 22(6) (2001), 885-893.

[25] R.E. Mrak and W.S. Griffin, Potential inflammatory biomarkers in Alzheimer's disease, J Alzheimers Dis 8(4) (2005), 369375.

[26] C.M. Norris et al., Calcineurin triggers reactive/inflammatory processes in astrocytes and is upregulated in aging and Alzheimer's models, J Neurosci 25(18) (2005), 4649-4658.

[27] P.H. Reddy et al., Gene expression profiles of transcripts in amyloid precursor protein transgenic mice: up-regulation of mitochondrial metabolism and apoptotic genes is an early cellular change in Alzheimer's disease, Hum Mol Genet 13(12) (2004), 1225-1240.

[28] M.V. Kolev et al., Implication of complement system and its regulators in Alzheimer's disease, Curr Neuropharmacol 7(1) (2009), 1-8.

[29] F.S. Shie et al., Modulation of microglial innate immunity in Alzheimer's disease by activation of peroxisome proliferatoractivated receptor gamma, Curr Med Chem 16(6) (2009), 643651.

[30] S.C. Tang et al., Toll-like receptor-4 mediates neuronal apoptosis induced by amyloid beta-peptide and the membrane lipid peroxidation product 4-hydroxynonenal, Exp Neurol 213(1) (2008), 114-121.

[31] J.K. Yao et al., Reduced membrane lipids in the cortex of Alzheimer's disease transgenic mice, Neurochem Res 34(1) 
(2009), 102-108.

[32] J. Zhou et al., Complement C3 and C4 expression in C1q sufficient and deficient mouse models of Alzheimer's disease, J Neurochem 106(5) (2008), 2080-2092.

[33] E.M. Blalock et al., Incipient Alzheimer's disease: microarray correlation analyses reveal major transcriptional and tumor suppressor responses, Proc Natl Acad Sci U S A 101(7) (2004), 2173-2178.

[34] D.M. Bonifati and U. Kishore, Role of complement in neurodegeneration and neuroinflammation, Mol Immunol 44(5) (2007), 999-1010.

[35] P.A. Desplats et al., Selective deficits in the expression of striatal-enriched mRNAs in Huntington's disease, $\mathrm{J} \mathrm{Neu}$ rochem 96(3) (2006), 743-757.

[36] A. Hodges et al., Regional and cellular gene expression changes in human Huntington's disease brain, Hum Mol Genet 15(6) (2006), 965-977.

[37] R. Inagaki et al., Omi / HtrA2 is relevant to the selective vulnerability of striatal neurons in Huntington's disease, Eur J Neurosci 28(1) (2008), 30-40.

[38] N.J. Maragakis and J.D. Rothstein, Mechanisms of Disease: astrocytes in neurodegenerative disease, Nat Clin Pract Neurol 2(12) (2006), 679-689.

[39] S. Sarkar and D.C. Rubinsztein, Small molecule enhancers of autophagy for neurodegenerative diseases, Mol Biosyst 4(9) (2008), 895-901.

[40] H. Wang et al., Nortriptyline delays disease onset in models of chronic neurodegeneration, Eur J Neurosci 26(3) (2007), 633-641.

[41] E. Bossy-Wetzel, A. Petrilli and A.B. Knott, Mutant huntingtin and mitochondrial dysfunction, Trends Neurosci 31(12) (2008), 609-616.

[42] K. Bossers et al., Analysis of gene expression in Parkinson's disease: possible involvement of neurotrophic support and axon guidance in dopaminergic cell death, Brain Pathol 19(1) (2009), 91-107.

[43] S. Mandel, E. Grunblatt and M. Youdim, cDNA microarray to study gene expression of dopaminergic neurodegeneration and neuroprotection in MPTP and 6-hydroxydopamine models: implications for idiopathic Parkinson's disease, J Neural Transm Suppl (60) (2000), 117-124.

[44] J.A. Olzmann et al., Selective enrichment of DJ-1 protein in primate striatal neuronal processes: implications for Parkinson's disease, J Comp Neurol 500(3) (2007), 585-599.

[45] F. Simunovic et al., Gene expression profiling of substantia nigra dopamine neurons: further insights into Parkinson's dis- ease pathology, Brain 132(Pt 7) (2009), 1795-1809.

[46] P. Teismann and J.B. Schulz, Cellular pathology of Parkinson's disease: astrocytes, microglia and inflammation, Cell Tissue Res 318(1) (2004), 149-161.

[47] M.A. Hauser et al., Expression profiling of substantia nigra in Parkinson disease progressive supranuclear palsy, and frontotemporal dementia with parkinsonism, Arch Neurol 62(6) (2005), 917-921.

[48] A. Bhathena and B.B. Spear, Pharmacogenetics: improving drug and dose selection, Curr Opin Pharmacol 8(5) (2008), 639-646.

[49] A.D. Roses et al., Complex disease-associated pharmacogenetics: drug efficacy, drug safety, and confirmation of a pathogenetic hypothesis (Alzheimer's disease), Pharmacogenomics J 7(1) (2007), 10-28.

[50] H.S. Hain, J.K. Belknap and J.S. Mogil, Pharmacogenetic evidence for the involvement of 5-hydroxytryptamine (Serotonin)-1B receptors in the mediation of morphine antinociceptive sensitivity, J Pharmacol Exp Ther 291(2) (1999), 444-449.

[51] S.D. Mague et al., Mouse model of OPRM1 (A118G) polymorphism has sex-specific effects on drug-mediated behavior, Proc Natl Acad Sci U S A 106(26) (2009), 10847-10852.

[52] A.H. Harrill et al., Population-based discovery of toxicogenomics biomarkers for hepatotoxicity using a laboratory strain diversity panel, Toxicol Sci 110(1) (2009), 235-243.

[53] M. Dillhoff, S.E. Wojcik and M. Bloomston, MicroRNAs in solid tumors, J Surg Res 154(2) (2009), 349-354.

[54] Y.S. Lee and A. Dutta, MicroRNAs in cancer, Annu Rev Pathol 4 (2009), 199-227.

[55] R. Saba et al., A miRNA signature of prion induced neurodegeneration, PLoS One 3(11) (2008), e3652.

[56] K. Wang et al., Circulating microRNAs, potential biomarkers for drug-induced liver injury, Proc Natl Acad Sci U S A 106(11) (2009), 4402-4407.

[57] N.L. Anderson and N.G. Anderson, The human plasma proteome: history, character, and diagnostic prospects, Mol Cell Proteomics 1(11) (2002), 845-867.

[58] K. Fujii et al., Multidimensional protein profiling technology and its application to human plasma proteome, J Proteome Res 3(4) (2004), 712-718.

[59] J.T. Lathrop et al., Therapeutic potential of the plasma proteome, Curr Opin Mol Ther 5(3) (2003), 250-257.

[60] H.J. Lee et al., Biomarker discovery from the plasma proteome using multidimensional fractionation proteomics, Curr Opin Chem Biol 10(1) (2006), 42-49. 


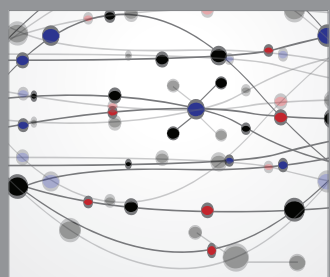

The Scientific World Journal
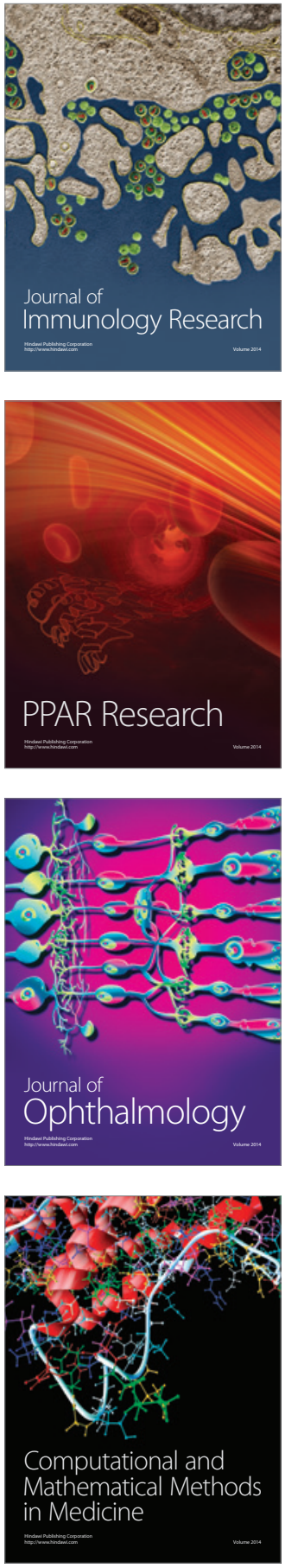

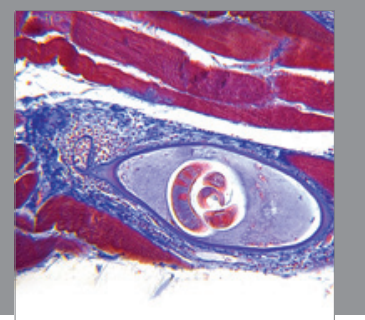

Gastroenterology

Research and Practice
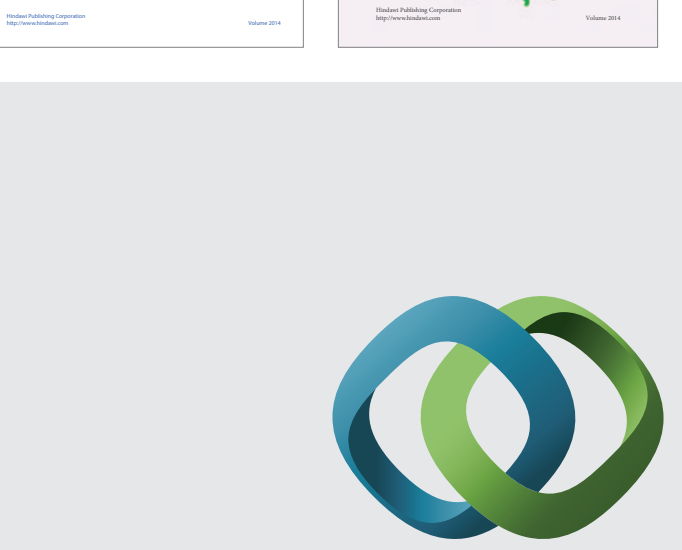

\section{Hindawi}

Submit your manuscripts at

http://www.hindawi.com
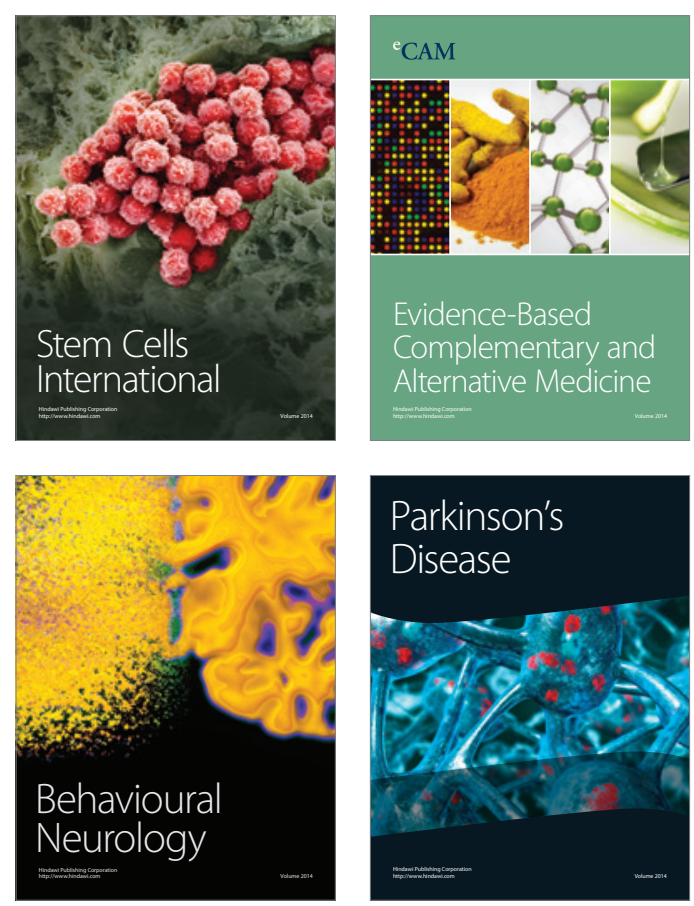

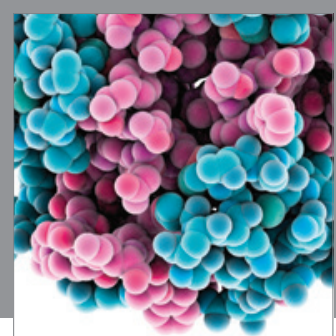

Journal of
Diabetes Research

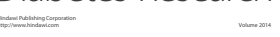

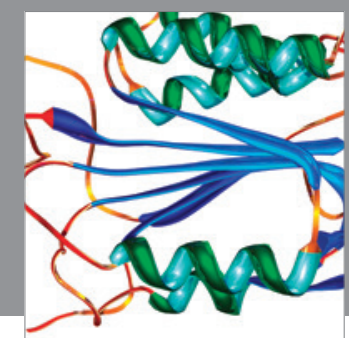

Disease Markers
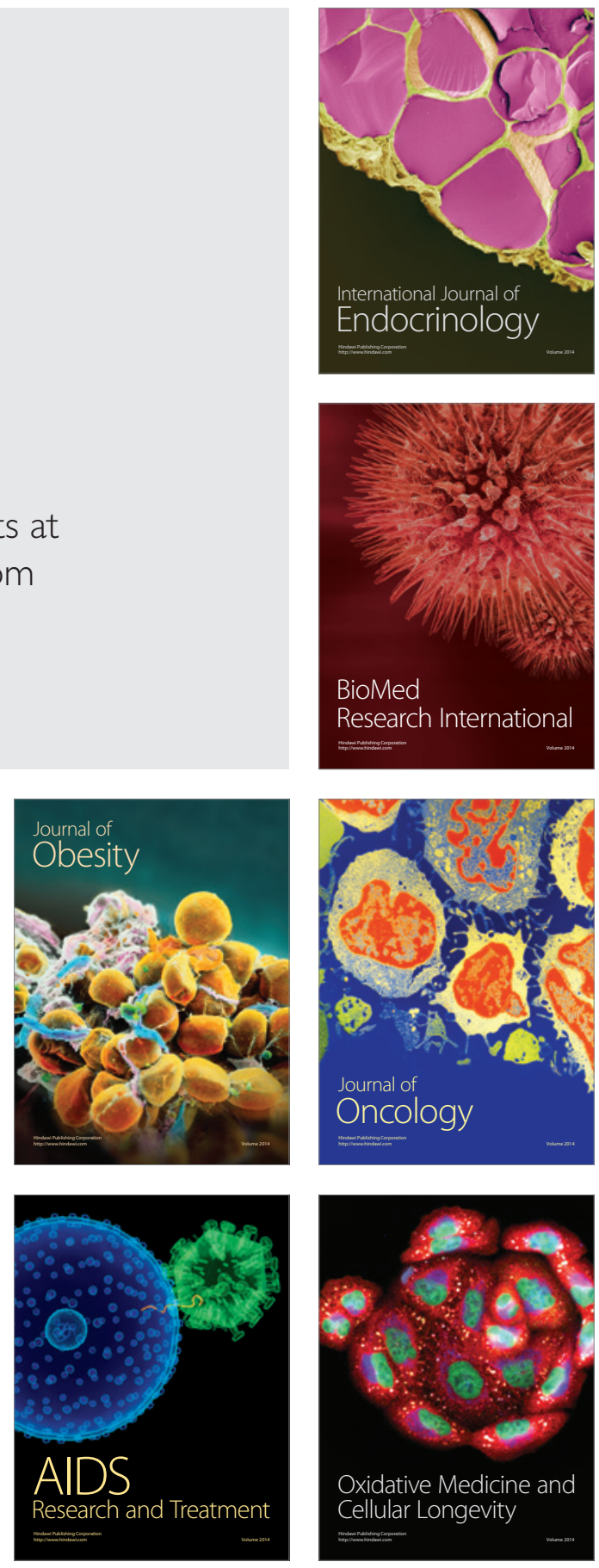